\title{
Corrigendum
}

\section{Cloning and Characterization of ifitm1 and ifitm3 Expression During Early Zebrafish Development - CORRIGENDUM}

Wei-Wei Xue, Huan-Nan Wang, Zhi-Meng Wang, Meng-Xi Qiu, Jing Che, Feng-Jiao Deng and Jiang-Dong Liu

doi:10.1017/S0967199414000756, Published by Cambridge University Press, 25 March 2015.

The authors apologise for errors in the corresponding authors details given on page 1 of the article. Below is the correct information of the corresponding author and email address :

1) Wei-Wei Xue, Huan-Nan Wang, Zhi-Meng Wang, Meng-Xi Qiu, Jing Che, Feng-Jiao Deng* and JiangDong Liu*

2)* All correspondence to: Feng-Jiao Deng and JiangDong Liu. e-mail: fish4@whu.edu.cn
3) All authors are from the same one laboratory. The second laboratory was superfluous and should be deleted.

\section{Reference}

Wei-Wei Xue, Huan-Nan Wang, Zhi-Meng Wang, Meng$X_{i}$ Qiu, Jing Che, Feng-Jiao Deng, \& Jiang-Dong Liu. Cloning and Characterization of ifitm1 and ifitm3 Expression During Early Zebrafish Development. Zygote. Published by Cambridge University Press, 25 March 2015. doi:10.1017/S0967199414000756. 